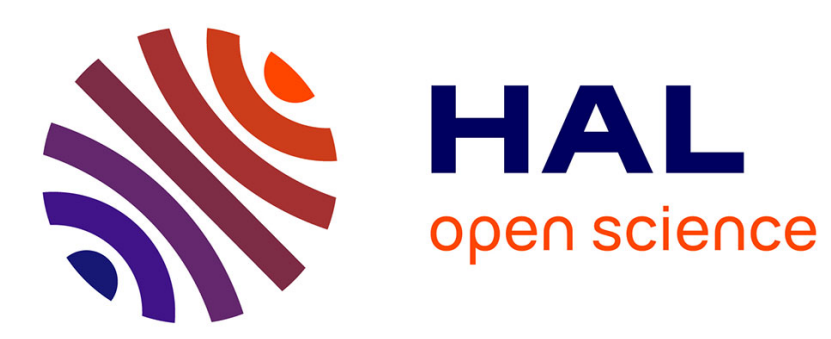

\title{
MICROSCOPIC THEORY OF MULTIFRAGMENTATION IN HIGH ENERGY HEAVY-ION COLLISIONS
}

\author{
A. Rosenhauer, Joerg Aichelin, H. Stöcker, W. Greiner
}

\section{- To cite this version:}

A. Rosenhauer, Joerg Aichelin, H. Stöcker, W. Greiner. MICROSCOPIC THEORY OF MULTIFRAGMENTATION IN HIGH ENERGY HEAVY-ION COLLISIONS. Journal de Physique Colloques, 1986, 47 (C4), pp.C4-395-C4-407. 10.1051/jphyscol:1986447 . jpa-00225813

HAL Id: jpa-00225813

https://hal.science/jpa-00225813

Submitted on 1 Jan 1986

HAL is a multi-disciplinary open access archive for the deposit and dissemination of scientific research documents, whether they are published or not. The documents may come from teaching and research institutions in France or abroad, or from public or private research centers.
L'archive ouverte pluridisciplinaire HAL, est destinée au dépôt et à la diffusion de documents scientifiques de niveau recherche, publiés ou non, émanant des établissements d'enseignement et de recherche français ou étrangers, des laboratoires publics ou privés. 


\title{
MICROSCOPIC THEORY OF MULTIFRAGMENTATION IN HIGH ENERGY HEAVY-ION COLLISIONS
}

\author{
A. ROSENHAUER, J. AICHELIN ${ }^{*}$, H. STÖCKER ${ }^{(1)}$ and W. GREINER \\ Institut für Theoretische Physik der J.W.Goethe-Universität \\ Frankfurt, D-6000 Frankfurt, F.R.G. \\ *Institut für Theoretische Physik Universität Heidelberg and \\ Max-Planck-Institut Heidelberg, D-6900 Heidelberg, F.R.G.
}

\begin{abstract}
We introduce a novel $\mathrm{N}$-body approach to describe heavy ion collisions. The nucleons are represented as Gaussian wave packets in configuration and momentum space. They interact via a combination of a Skyrme type potential and a Uehling-Uhlenbeck two-body scattering mechanism. This theory keeps track of the correlations and therefore allows for a selfconsistent theoretical description of the entire complex dynamics of heavy ion collisions, from the initial non-equilibrium penetration stage via the high density phase to the final formation of complex-stable and instable-fragments. The cluster formation is caused by correlations and density fluctuations. We find that global equilibrium is not established in the course of the reaction. The form of the mass yield curve agrees with experimental findings. We also study the recently observed collective flow effects and find a strong dependence on the nuclear equation of state.
\end{abstract}

The current experimental and theoretical interest in high energy heavy ion collisions is concentrated on two topics: first, one would like to infer the properties of nuclear matter at high density and temperature which are probed in the early states of a heavy ion reactions ${ }^{1}$ Of particular interest are the nuclear equation of state and the transport coefficients. The knowledge of the nuclear equation of state is of great importance for an understanding of the early universe, for the collapse of supernovae and for the stability of neutron stars.

Secondly, one would like to study the behaviour of highly excited nuclear matter at $10 \mathrm{w}$ densities ${ }^{2}$. This is realized in the final disassembly state of the reactions. Experimentally several intermediate mass clusters are observed ${ }^{3}$. This has been interpreted both as a signature of a liquid vapour phase transition ${ }^{4}$ and as a nonequilibrium statistical break-up of cold matter ${ }^{5}$.

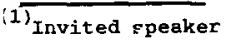


Up to now a theory which describes simultaneously both the creation of the high density matter and the formation of complex fragments is not at hand: The macroscopic nuclear fluid dynamical (NFD-)model has first exhibited the relation between the equation of state and the collective flow and pion production ${ }^{1,6}$. The main disadvantage of the NFD-model is, however, that much of the microscopic dynamics is 1ost; in particular are the initial nonequilibrium state of the reaction and the fragment formation outside of the range of applicability of this approach.

The most advanced microscopic theory developed so far is the Vlasov-Uehling-Uhlenbeck theory. This has been applied successfully to describe compression phenomena such as the collective sidewards flow ${ }^{7}$ and transverse momentum transfer ${ }^{8}$, pion $^{7}$ - and strangeness ${ }^{9}$ production, and gives their dependence on the stiffness of the equation of state. Furthermore, nonequilibrium (finite mean free path) and quantal effects such as partial transparency in light systems, the importance of Pauli blocking for nuclear stopping and linear momentum transfer have been studied 10 .

However, the numerical method used to solve the VUJ equations impose an ensemble averaging to calculate smooth single particle distribution functions necessary to evaluate the local density dependent mean field $U(\rho)$ as well as the (l-f) (l-f) Pauli blocking probabilities.

Therefore, both the NFD and the VUU approach cannot address the mu1tifragmentation. They are limited to the describe one-body observables only while the fragmentation process involves many-body correlations.

A large number of models has been introduced to describe the formation of complex fragments in the late stage of the reaction. Most of these models are static calculations or are statistical in nature, thus neglecting completely the dynamical evolution of the collision $2,4,5$. Other approaches use a simplified model for the dynamics of the expansion ${ }^{11}$. They are unable to treat the early state of the reaction and rely on assumptions about the configuration at the state of highest den- 
sity. The latter models emphasize the role of the fluctuations for fragment formation.

There is one exception to this classification of models, namely the classical "Molecular Dynamics" (MD) or "equation of motion" approach, which - in principle - is capable of treating consistently both the compression and the fragments formation, albeit on a completely classical leve1 : Hamilton's equations of motion are integrated for $\mathrm{N}$ classical point particles with finite range $n-n$ potentials, which are adjusted to give reasonable $n-n$ scattering cross sections and nuclear binding properties $^{12}$. As a result of the implemented hard core repulsion, the classical MD approach predicts a collective sidewards flow in qualitative agreement with the data. It incorporates the complete classical $\mathrm{N}$-body dynamics, which is necessary to describe the formation of clusters. This aspect of the classical MD approach has been studied up to now only in a simple scenario ${ }^{13}$.

Here we present a novel approach, based on the MD method, which explicitly incorporates $\mathrm{N}$-body correlations, a nuclear equation of state and the most important quantum corrections, namely the Pauli principle, stochastic scattering, and particle production.

Hence the present approach combines the advantages of the classical molecular dynamics approach (explicit two-body interaction) with the important quantum features, which - as we know from vUU calculations - are essential to describe the reaction dynamics properly.

We have therefore dubbed our approach "quantum molecular dynamics", QMD, to distinguish it from the purely classical "molecular dynamics" methods well known from the literature.

Each nucleon is represented by a Gaussian Wigner density in momentum and coordinate space. The initial centroids of position and momentum of $A_{T}+A_{P}$ wavepackets are not assigned randomly but chosen judiciously to respect ab initio the pauli principle : in contrast to cascade and vu calculations we do only accept configurations in which two nucleons have at least a distance of $1.5 \mathrm{~h}$ in phase space. Only about 1 out of 
200 randomly chosen configurations fulfills this requirement. This is accomplished by first assigning the positions of the centroids of the $A_{P}$ $+\mathrm{A}_{\mathrm{T}}$ wave packets randomly, with a minimum distance of 1.5 fm between the centers of each pair of wave packets. Then the centroid of the momentum of each wave packet is assigned randomly between zero and $P_{F}(r)$. $\mathrm{P}_{\mathrm{F}}(x)$ is obtained via the Thomas-Fermi model from the local density, which is given by the sum over the $A_{T}+A_{P}$ Gaussian wave packets in conEiguration space.

Every wave packet interacts simultaneously with every other wave packet via two- and three-body potentials of the Skyrme-type:

$u_{123}=t_{1} \delta\left(x_{1}-x_{2}\right)+t_{1} \delta\left(x_{2}-x_{3}\right)+t_{1} \delta\left(x_{1}-x_{3}\right)+t_{2} \delta\left(x_{1}-x_{2}\right) \delta\left(x_{1}-x_{3}\right)$

The total interaction potential felt by particle $j$ whose centroid is located at position $x$ is then given by

$U_{j}(x) \approx t_{1} \sum_{\substack{i=1 \\ i \neq j}}^{A_{T} T^{+A_{P}}} e^{-\left(x-x_{0 i}\right)^{2} / L}+t_{2}\left[\sum_{\substack{i=1 \\ i \neq j}}^{A_{1}+A_{P}} e^{-\left(x-x_{0 i}\right)^{2} / L}\right]^{2}$

With $\mathrm{L}$ given by $4.33 \mathrm{fm}^{2}$ for the presented calculations. This can easily be extended to incorporate Coulomb energy and velocity dependent forces. The term $\Sigma$ is proportional to the density. This allows to parametrize the total interaction potential in terms of the density and to relate the nn interaction with the nuclear equation of state which can be parametrized as :

$U=\alpha \rho+\beta \rho^{\gamma}$

Here $\alpha=-356(-124) \mathrm{MeV}, \beta=303(70.5) \mathrm{MeV}, \gamma=7 / 6(2)$ defines the medium (stiff) equation of state, respectively, which have been used extensively in earlier VUU calculations.

We let the Gaussian wave packets propagate under the influence of 
the (N-1)body interactions with the respective other Gaussians, following the trajectory of the centroid with a second order integration method. A timestep of $0.1 \mathrm{fm} / \mathrm{c}$ is used. The width of the wave packets is - in the first calculation presented below - kept constant in time to reduce the numerical expenditure.

Two wave packéts can also scatter stochastically and produce deltas and pions. For the exploratory calculations presented below, scattering occurs at the distance of closest approach if the centroids of two Gaussian wave packets approach each other to within $d=\sqrt{\sigma 7 \pi}$ in analogy to the vUU approach. The experimental $\sigma(\mathrm{nn})$ parametrization is taken from ref. 14. We are presently working on an explicit stochastic treatment of the uncertainty principle using positions and momenta according to the probability distribution given by the Gaussian width of the wave packets, thus dropping the exact conserving energy and momentum for individual nn collisions.

The scattering is Pauli blocked with a probability ( $1-f)(1-f)$, where $f$ is the finite occupation of the phase space cell into which the centroid of the Gausian would be scattered. The phase space cells at the nuclear surface include some of the surrounding vacuum; this excess phase space is substracted if it is classically forbidden. The evolution of single nuclei has been followed for a time span of $200 \mathrm{fm} / \mathrm{c}\left(6 \times 10^{-22}\right.$ sec). This is considerably longer than the typical collision times at medium and high energies. We observe a surprisingly stable density distribution, which is actually even more stable than the corresponding vUJ calculation.

After checking the stability of the initial nuclei, we proceed to study the effects of the $\mathrm{N}$-body correlations in nuclear collisions at high energy.

For this purpose the two nuclei are boosted towards each other with the appropriate velocity of the equal speed system.

Fig. 1 shows a calculation for the system $\mathrm{Nb}(0.4 \mathrm{GeV} /$ nucleon, $b=$ $3 \mathrm{fm})+\mathrm{Nb}$. We study whether the QMD-approach predicts correctly the re- 
cently observed flow ${ }^{1,15}$.

In the top (bottom) row we see the final configuration space distribution of one typical event, the final momentum space distribution of the same event, and an overlay of the final momentum distribution of ten events using the medium (stiff) equation of state, respectively. The stiffer the equation of state the larger is the flow angle. This prediction of QMD agrees with previous VUU calculations. ${ }^{7,8}$. The QMD approach predicts in addition to the sidewards flow the formation of clusters, which cannot be studied directly in the vU theory. It will be interesting to see whether the complex fragments exhibit a more sharply peaked flow distribution than the proton dominated average flow per event, as was predicted by fluid dynamical calculations ${ }^{1}$. This is presently also under experimental investigation with the upgraded GSI/LBL Plastic Ball $4 \pi$ spectrometer system ${ }^{15}$, which is now able to identify and measure all outgoing fragments from pions to Neon nuclei in high multiplicity events.

Figure 2 shows three typical collisions of $800 \mathrm{MeV} / \mathrm{n} \mathrm{Ne+Au,} \mathrm{two} \mathrm{of}$ them at $b=0 \mathrm{fm}$ and one at $b=6 \mathrm{fm}$. For four different times $(t=50$ $\mathrm{fm} / \mathrm{c}, 100 \mathrm{fm} / \mathrm{c}, 150 \mathrm{fm} / \mathrm{c}, 180 \mathrm{fm} / \mathrm{c})$ the centroids of the wave packets are projected onto the scattering plane. The centroids of the wave packets are marked by circles of a radius of $r=3 \mathrm{fm}$.

We define two nucleons as members of a complex fragment if the distance of their centroids in coordinate space is smaller than 3 fm at $t=$ $180 \mathrm{fm} / \mathrm{c}$. Therefore, in $\mathrm{fig} .2$ the overlapping define directly the clusters.

The initial high density penetration stage is completed at about $t$ $=50 \mathrm{fm} / \mathrm{c}$. Here some of the projectile nucleons escape from the interaction region in the forward direction. In addition some low energy particles, which have suffered at least one scattering, are emitted sidewards. The most intriguing observation is, however, the formation of several stable fragments of medium masses, which are best seen at later times. These are formed as a result of the explicit $\mathrm{N}$-body correlations 
of the present QMD approach. In VUU calculations one large remnant can be seen at depleted density 8,10 . The local density fluctuations, which are responsible for the formation of clusters, do not occur in a mean field calculations (e.g. TDHF, VUU) since the ensemble averaging smears out the density fluctuations and consequently the mean potential forms a contineous low density region.

The present approach allows also for the study of the fluctuations in the collisions: even for the same impact parameter we see very dif. ferent final states as far as the formation of clusters is concerned. The first column shows an event (event 8) in which several large clusters of roughly equal size are formed. In the second column (event 9) only one large cluster and one small cluster are seen. In addition many light fragments $A \leq 3$ are emitted.

Fig. 3 shows the calculated mass yield distribution for central collisions. We would like to point out that the general trend of $Y(A)$ agrees with experimentally observed fragment yields ${ }^{3}, 4$. Better statistics must be accumulated, of course, before a detailed comparison with the data is feasible.

It is particularly intriguing that the effects of the nuclear potential energy on the flow angular distributions, fragment yields and spectra can be studied with the QMD approach.

In conclusion we have introduced a new approach to study $\mathrm{N}$-body correlations in heavy ion collisions which goes beyond the classical level. The nucleons are represented by Gaussian wave packets in configuration and momentum space. They interact via an effective skyrme force and two-body collisions. The Pauli principe is obeyed throughout the collisions, both in the initial state (by construction) and also in the course of the reaction. Here the stochastic nn scattering is Pauli suppressed with a probability given by the final state phase space occupancy. We construct reasonably stable nuclei to study heavy ion colli. sions. We observe fast particle emission and the formation of several 
complex fragments per event in violent collisions of $\mathrm{Ne}(0.8 \mathrm{GeV} / \mathrm{Nucleon})$ $+\mathrm{Au}$ and $\mathrm{Fe}(0.2 \mathrm{GeV} /$ nucleon $)+\mathrm{Au}$.

The theory does not only give the yields and spectra of intermediate mass fragments, but also predicts the recently observed collective sidewards flow. This novel approach can well be applied also at much lower energies, e.g. to study multifragmentation events, and is particularly suitable to study the influence of the nuclear potential.

\section{References:}

1 H. Stöcker and W. Greiner, Phys. Rep. in press

R. Stock, Phys. Rep. in press

2 J. Hüfner, Phys. Rep. $\underline{125}\left(1985_{i}\right) 129$

L. Csernai and J. Kapusta, Phys. Rep. 131(1986)223

3 B. Jakobsson, Z. Phys. A 307 (1982) 293

C.J. Waddington and P.S. Freier, Phys. Rev. C31(1985)888

A.I. Warwick, H.H. Wiemann, H.H. Gutbrod, M.R. Maier, J. Peter, M. Freedman, D.J. Henderson, S.B. Kaufman, E.P. Steinberg and B.D. Wilkins, Phys. Rev. C27(1983) 1083

4 A.S. Hirsch, A. Bujak, J.E. Finn, L.J. Gutay, R.W. Minich, N.T, Porile, R.P. Scharenberg, B.C. Stringfellow and F. Turkot, Phys. Rev. C29 (1984) 508

A.D. Panagiotou, M.W. Curtin, H. Toki, D.K. Scott and P.J. Siemens, Phys. Rev. Lett. 52(1984)496

H. Stöcker, G. Buchwald, G. Gräbner, J. Theis, J.A. Maruhn and W. Greiner, Nuc1. Phys. $\underline{400}$ (1983)63

$5 \mathrm{~J}$. Aichelin, J. Hüfner and R. Ibarra, Phys. Rev. C30(1984)107

$\mathrm{X}$. Campi, J. Desbois and E. Lipparini, Phys. Lett. 142B(1984)8

6 H. Stöcker, W. Greiner and W. Scheid, Z. Phys. A286(1978) 121

H. Stöcker, J. Maruhn and W. Greiner, Z. Phys. A290(1.979) 297

7 H. Kruse, B.V Jacak and H. Stöcker, Phys. Rev. Lett. 54 (1985) 289

8 J.J. Molitoris and H. Stöcker, Phys. Rev. C32(1985)346 and Phys. Lett. $156 \mathrm{~B}(1985)$

9 J. Aichelin and C.M. Ko, Phys. Rev. Lett. 55(1985)2661

$10 \mathrm{~J}$. Aichelin and H. Stöcker, Phys. Lett. 163B(1985)59

J. Aichelin, Phys. Rev. C33(1986) 537

11 J. Knoll and B. Strack, Phys. Lett. 149B (1984)45

3. Knoll, Proceedings of the Topical Meeting on Phase Space Approach to Nuclear Dynamics, Trieste Sept. 85, World Scientific Publisching, Singapore

12 A.R. Bodmer, C.N. Panos and A.D. Mackellar, Phys. Rev. C22(1980)1025

L.Wilets, Y. Yariv, R. Chestnut, N.P. A301(1978) 359

J.J. Molitoris, J.B. Hoffer, H. Kruse, H. Stöcker, Phys. Rev.lett. $\underline{53}(1984) 899$ 
13 R.J. Lenk and V.R. Pandharipande, Preprint University of Illinois A. Vicentini, G. Jacucci and V.R. Pandharipande, Phys, Rev. C31 (1985) 1783

14 J. Cugnon, Phys. Rev. C22(1980)1885

15 H.G. Ritter, Nucl. Phys. 3C(1985)A447

H.A. Gustafsson, H.H. Gutbrod, B. Kolb, H. Löhner, B. Ludewigt, A.M. Postkanzer, T. Renner, H. Riedesel, H.G. Ritter, A. Warwick and H. Wieman, Phys. Lett. 142B(1984)141

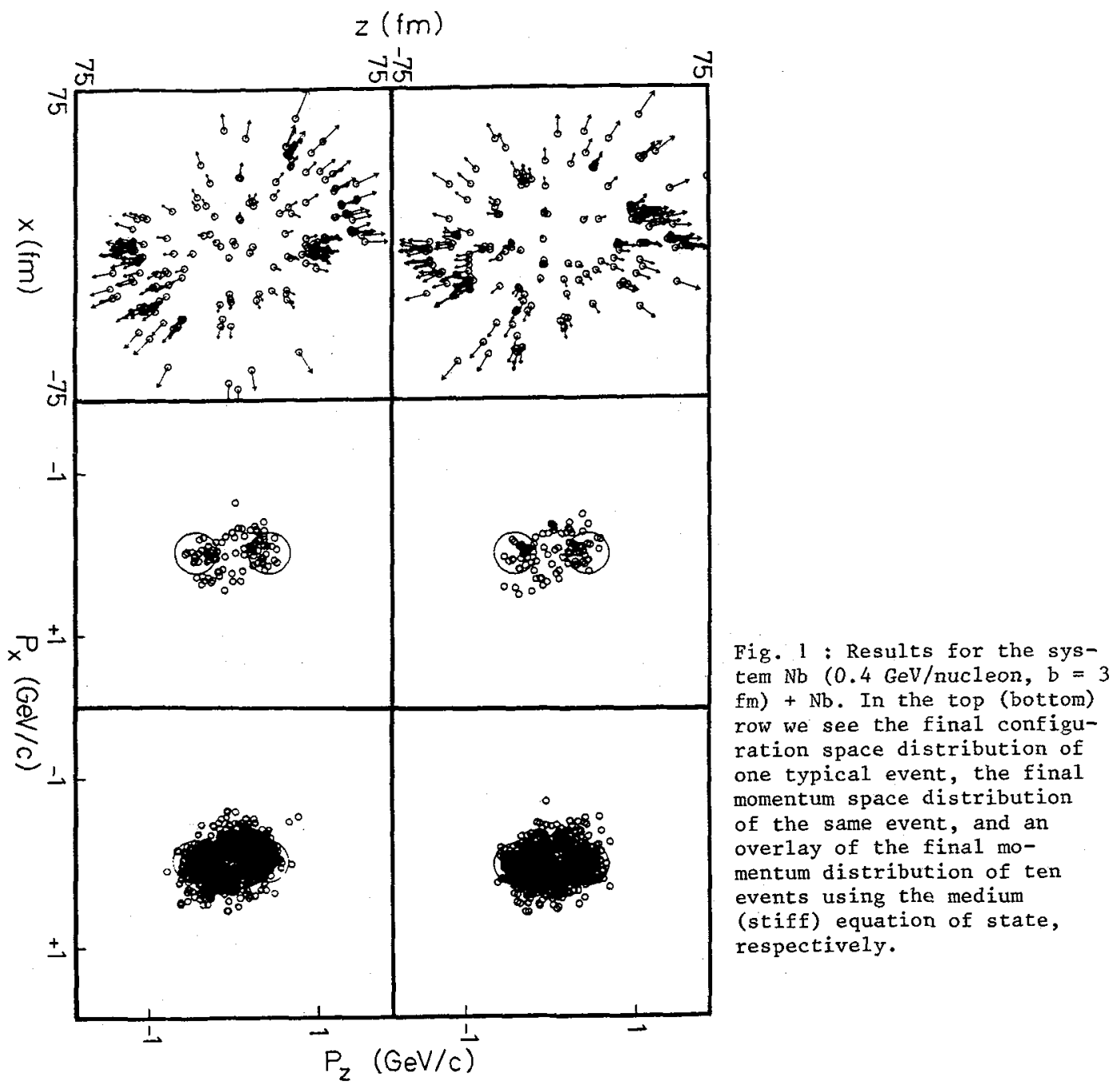




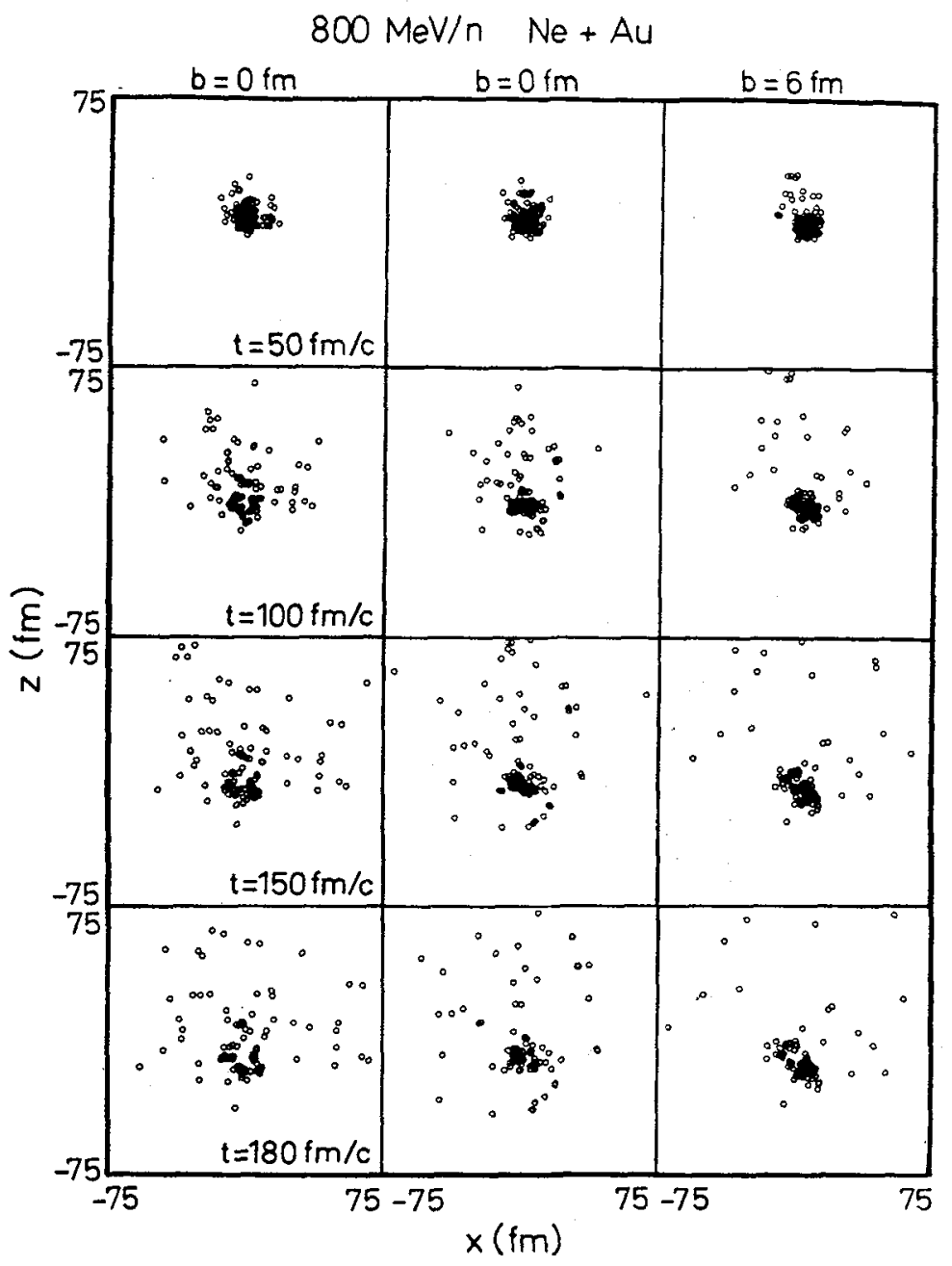

Fig. 2 : Three typical collisions of $800 \mathrm{MeV} / \mathrm{n} \mathrm{Ne}+\mathrm{Au}$, two of them at $\mathrm{b}=$ $c \mathrm{fm}$ and one $a t b=6 \mathrm{fm}$. For four different times ( $t=50$ $\mathrm{fm} / \mathrm{c}, 100 \mathrm{fm} / \mathrm{c}, 150 \mathrm{fm} / \mathrm{c}, 180 \mathrm{fm} / \mathrm{c}$ ) the centroids of the wave packets are projected onto the scattering plane. The centroids of the wave packets are marked by circles of a radius of $r=3$ fm. 


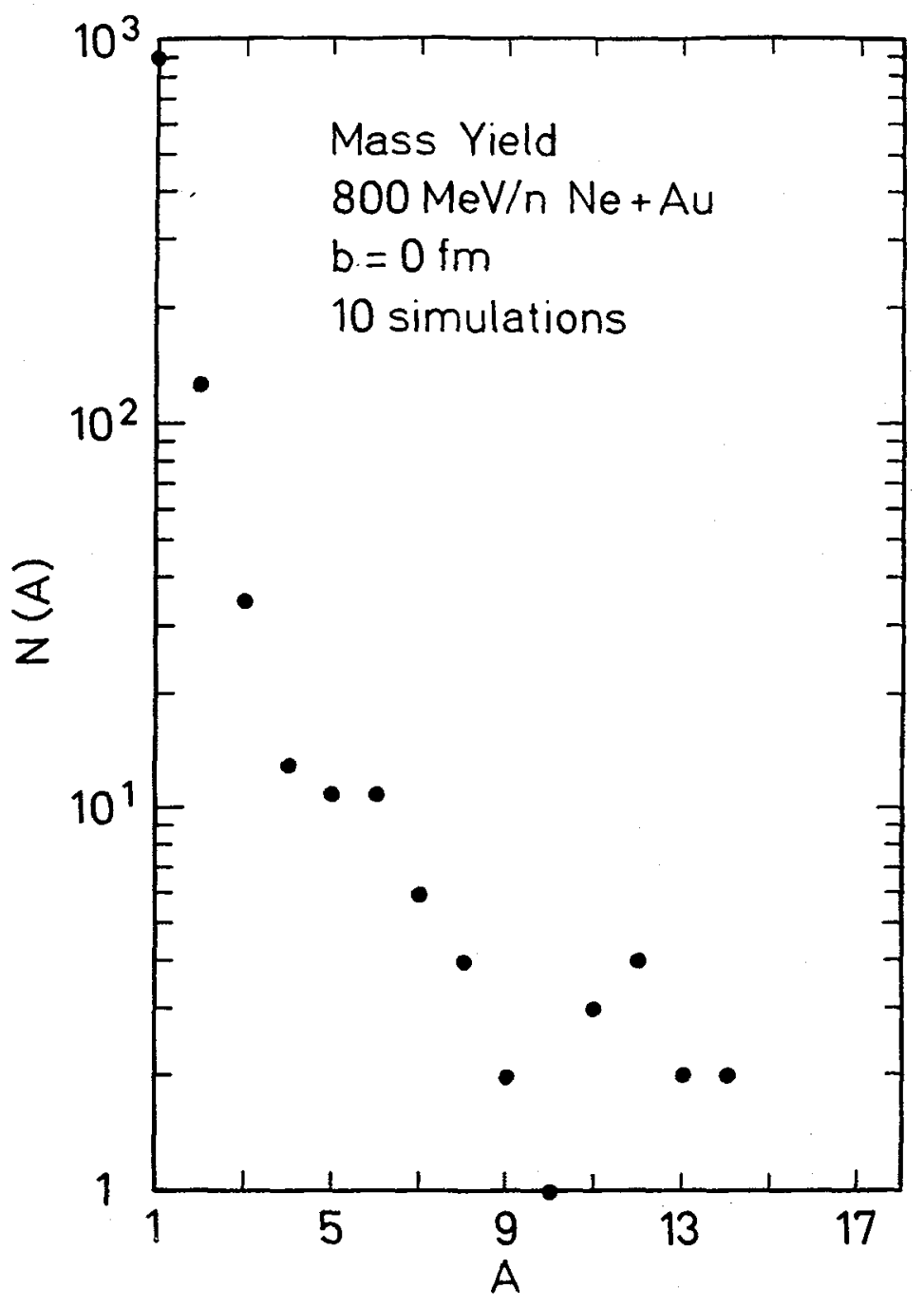

Fig. 3: Calculated mass yield distribution for central collisions of the system $800 \mathrm{MeV} /$ nucleon $\mathrm{Ne}+\mathrm{Au}$. 

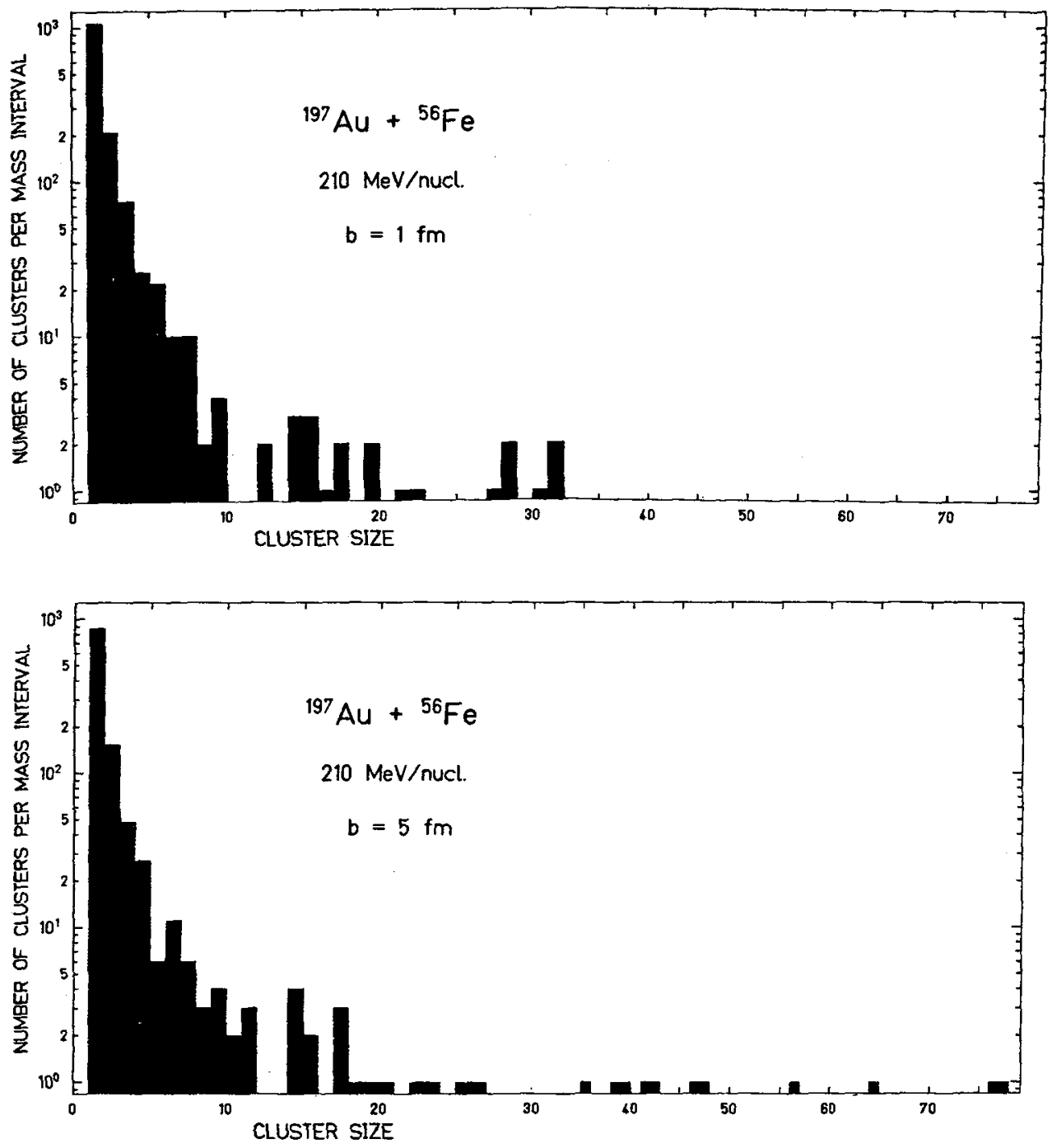

Fig. 4: Mass yield distribution for the reaction $210 \mathrm{MeV} /$ nucleon $\mathrm{Fe}+\mathrm{Au}$ at impact parameters of $\mathrm{b}=1$ and $\mathrm{b}=5 \mathrm{fm}$ as indicated. 
RAPIDITY DISTRIBUTION FOR 870 CLUSTERS OF MASS I RAPIDITY DISIRIBUTION FOR 1072 CLUSTERS OF MASS 1

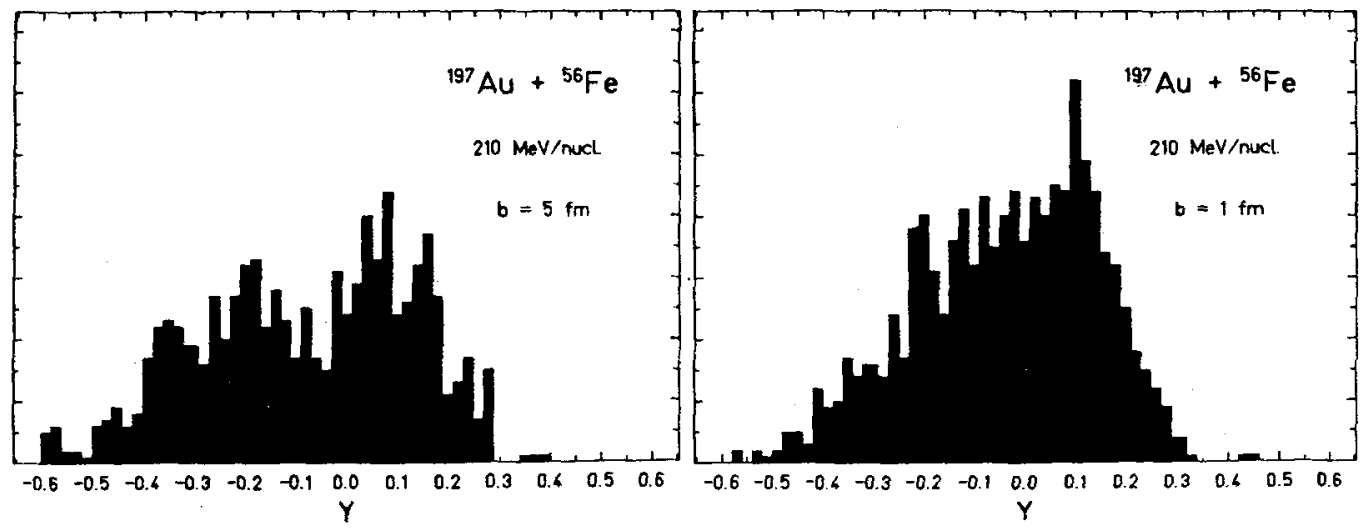

RAPIDITY DISTRIBUTION FOR 228 CLUSTERS OF MASS 2-4 RAPIDITY DISTRIBUTION FOR 311 CLUSTERS OF MASS 2-4

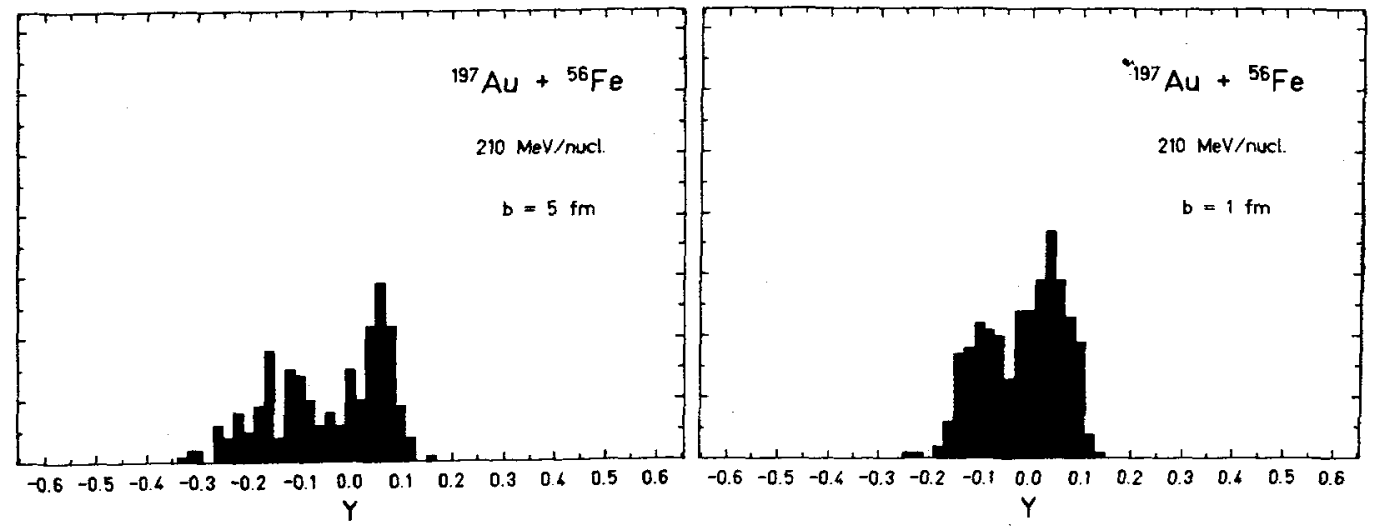

RAPIDITY DISTRIBUTION FOR 32 CLUSTERS OF MASS 5-10RAPIDITY DISIRIBUTION FOR 48 CLUSTERS OF MASS 5-10

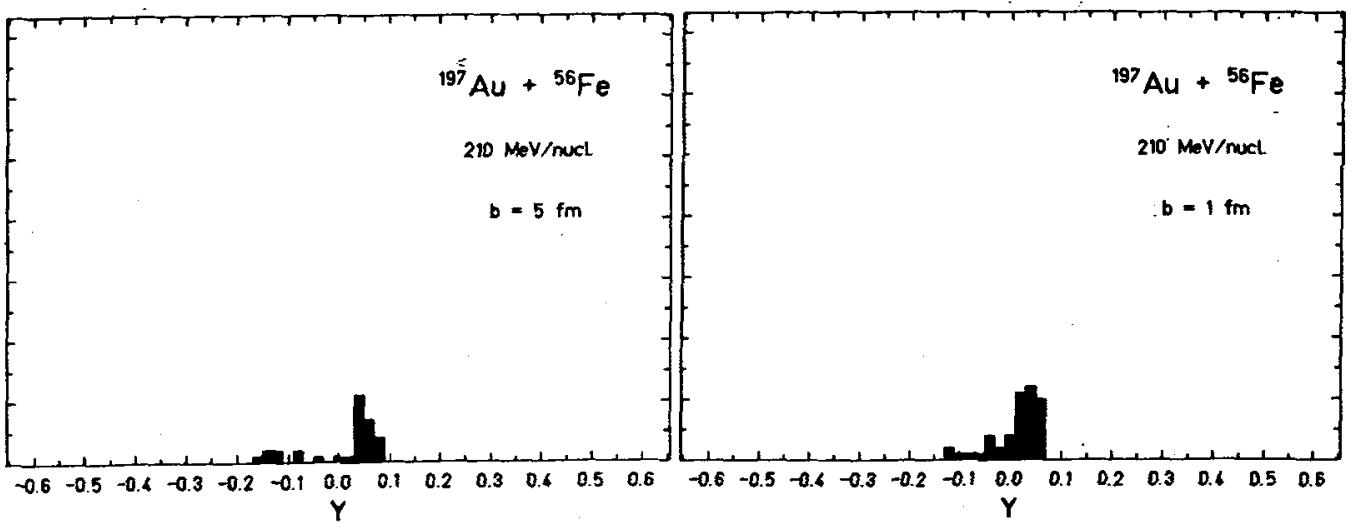

Fig. 5: Rapidity distribution $d N / d y$ for clusters of mass $1,2-4$ and 5-10 at intermediate impact parameters $(b=5 \mathrm{fm})$ and at almost central collisions $(b=1 \mathrm{fm})$ of the system $\mathrm{Fe}+\mathrm{Au}$ at $210 \mathrm{MeV} /$ nucleon. 